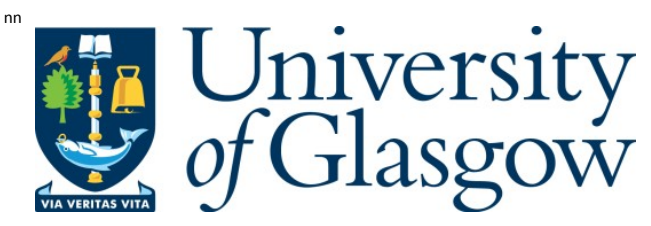

Big-A labo, A ., Harrison, P., and Cartmell, M . P. (2015) Contact model for elastoplastic analysis of half-space indentation by a spherical impactor. Computers and Structures, 151. pp. 20-29.

Copyright @ 2015 Elsevier, Ltd.

A copy can be downloaded for personal non-commercial research or study, without prior permission or charge

Content must not be changed in any way or reproduced in any format or medium without the formal permission of the copyright holder(s)

http://eprints.gla.ac.uk/102191/

Deposited on: 03 February 2015

Enlighten - Research publications by members of the U niversity of Glasgow http://eprints.gla.ac.uk 


\title{
CONTACT MODEL FOR ELASTOPLASTIC ANALYSIS OF HALF-SPACE INDENTATION BY A SPHERICAL IMPACTOR
}

\author{
${ }^{1}$ Akuro Big-Alabo ${ }^{a}$, Philip Harrison ${ }^{a}$, and Matthew P. Cartmell ${ }^{b}$ \\ ${ }^{a}$ Systems, Power and Energy Research Division, School of Engineering, University of \\ Glasgow, G12 8QQ, Scotland, UK \\ ${ }^{\mathrm{b}}$ Department of Mechanical Engineering, University of Sheffield, Mappin Street, Sheffield, \\ S1 3JD, England, UK
}

\begin{abstract}
This paper presents a new contact model for analysis of post-yield indentation of a halfspace target by a spherical indenter. Unlike other existing models, the elastoplastic regime of the present model was modelled using two distinct force-indentation relationships based on experimentally and theoretically established indentation characteristics of the elastoplastic regime. The constants in the model were derived from continuity conditions and indention theory. Simulations of the present model show good prediction of experimental data. Also, an approach for determining the maximum contact force and indentation of an elastoplastic half-space from the impact conditions has been proposed.
\end{abstract}

Keywords: contact model, indentation, elastoplastic, plastic deformation, half-space, impact.

\footnotetext{
${ }^{1}$ Corresponding author - E: a.big-alabo.1@research.gla.ac.uk; T: +44 7774279617
} 


\section{Introduction}

Contact phenomenon finds application in many impact processes such as forging, stamping, shot-peening, and impact of projectiles on structures. Of primary interest are cases that involve plastic deformation and/or damage. Contact models that account for the effects of plastic deformation and/or damage are needed to facilitate analytical studies of post-yield impact events, to validate finite element models, and to validate indentation test results. In this study, a simple contact model that accounts for plastic deformation is formulated to model the indentation of a metallic target by a spherical indenter.

Impact is a dynamic phenomenon that involves two bodies coming in contact at a relative velocity. Generally speaking, the impact between two solid bodies gives rise to a localised deformation of the contacting bodies at the region of contact. Either both bodies are deformed locally or one of the bodies is deformed locally depending on the relative rigidity of the bodies. As a result of the occurrence of local deformation during impact between two solid bodies, the theoretical modelling of such impact events combines the contact mechanics of the localised deformation with the equations of motion of the contacting bodies. The contact mechanics for rate-independent impact response is developed based on static conditions and it gives the relationship between the force at the point of contact and the localised deformation of the contacting bodies. The sum of the local deformation of each of the contacting bodies is called the indentation or relative approach and this indentation is directly related to the contact force.

In several previous experimental investigations and for many practical impact problems, the conditions are such that one of the contacting bodies deforms locally and is relatively large compared to the other body. The latter is often stiff enough to withstand significant local deformation and can be considered to be completely rigid. The body that deforms locally is called the target and the rigid body is called the indenter, impactor or projectile. This kind of impact problem is referred to as a half-space impact and it is depicted in Figure 1 for the case of elastoplastic impact. It is customary to develop contact models for rate-independent impact analysis based on half-space conditions [1]. For instance, the Hertz contact theory gives the force-indentation relationship for the impact of a spherical indenter on an elastic half-space. A detailed treatment of the Hertz contact theory for different contacting surfaces can be found in [1, 2].

The Hertz contact theory is only applicable for small indentations where the impact is elastic. If the applied contact force is high enough so that the stress generated in the contact zone goes beyond the yield point, then plastic deformation occurs and significant deviations from Hertz contact theory are observed [3]. It has been shown that plastic deformation can occur at impact velocities as low as $0.14 \mathrm{~m} / \mathrm{s}$ [1]. Moreover, most practical impact events are characterised by plastic deformation [1, 2], and therefore it is necessary to account for plastic deformation in the contact model. The effect of plastic deformation is to reduce the contact force from that predicted by purely elastic deformations, and to produce a permanent indentation at the end of the contact, thereby, making the unloading path to be different from that of the loading. 
(a)
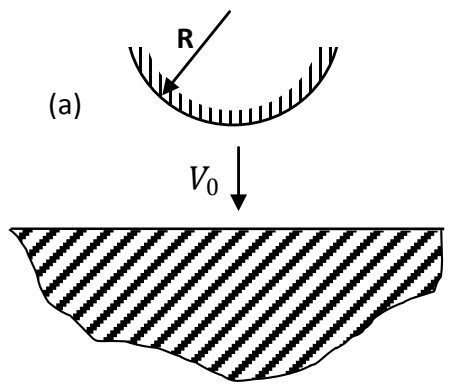

(b)

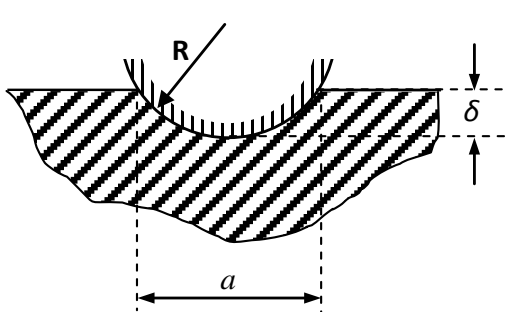

(c)
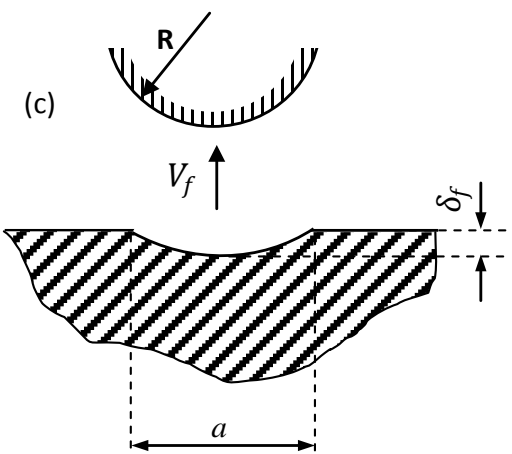

Figure 1: Elastoplastic half-space impact of a rigid spherical indenter on a compliant flat target (a) before impact (b) during impact (c) after impact.

Contact models that account for plastic deformation have been developed for metallic targets $[1,2,5,6,8]$ and for composite laminate targets [4, 7]. These models can be divided into two groups, namely: those that are developed based on two distinct loading regimes $[2,4,7]$, and those that are developed based on three loading regimes $[1,5,6,8]$. With metallic targets, three loading regimes (elastic, elastoplastic, and fully plastic) and an unloading regime are required for complete description of the contact mechanics $[1,5]$. For a contact model that is based on elastic-elastoplastic-fully plastic loading regimes, the loading features and force-indentation relationships for the elastic and fully (perfectly) plastic loading regimes are well established. The elastic loading regime starts from the beginning of the loading and ends at the yield point, where the mean contact pressure is given as $P_{0}=1.1 S_{y}$ ( $S_{y}$ is the yield strength of the target). The elastic loading regime can be modelled accurately using the well-known Hertz force-indentation relationship. The fully plastic loading regime is characterised by a linear force-indentation relationship and a constant mean pressure of $P_{0}=2.8 S_{y}$, which is equal to the Brinell hardness of the target [8]. The onset of the fully plastic regime occurs at the point where the mean pressure is $2.8 S_{y}$. The main difficulty lies in modelling the elastoplastic loading regime which starts at the yield point and ends at the onset of fully plastic conditions.

$\mathrm{Li}$ and co-workers [6] developed an elastoplastic contact model that is based on three loading regimes. The onset of the elastoplastic loading regime was proposed to lie at a mean pressure of $P_{0}=1.6 S_{y}$ and this elastoplastic loading regime was modelled using an equivalent ratio power relationship. The power law has three constants that depend on the material properties of the contact system and experimental data. However, the constants were determined from FEA results for the particular case considered. Also, the restitution model used in the study does not guarantee a smooth transition from the loading to the unloading phase. A more rigorous and recent contact model that captures plastic deformation effect and is based on three loading regimes has been provided by Brake [8], who modelled the elastoplastic loading regime by enforcing continuity between the yield point and the onset of fully plastic loading using a cubic Hermite interpolation polynomial. However, it was observed that the interpolation used in the elastoplastic regime produced an unrealistic bend sometimes. To obtain a more realistic fit, the onset of the fully plastic 
regime was moved from the initial point to a point where the slope of the fully plastic regime is parallel to the slope of the elastic regime. A restitution model that determines the unloading path based on the loading regime from which the unloading began was also developed. The restitution model provides a smooth transition from the loading to the unloading. A drawback of Brake's model for the elastoplastic loading regime is that it requires much more computational effort compared to the other models $[1,4,5,7]$.

In this paper, a new contact model that accounts for plastic deformation effects during the loading and restitution phases of the contact force is presented. The present study is motivated by (i) the gaps found in the attempts by past investigators to model the post-yield loading and restitution phases of a contact force, and (ii) the need to provide a simple and yet reasonably accurate contact model that can be easily used for impact modelling. The contact model is based on three loading regimes consisting of elastic, elastoplastic, and fully plastic regimes, and a single-phase restitution that is elastically nonlinear. Furthermore, the elastoplastic loading regime is divided into two distinct regions, and each region is modelled with a different force-indentation relationship that depicts the deformation mechanism observed from experiments and FEA results. A sketch of the compliance curve for the new contact model is shown in Figure 2. The structure of the remainder of this paper is as follows. In section 2, the force-indention models for the loading regimes and the unloading are presented, and the constants in these models are derived theoretically and from continuity conditions. In section 3 , the normalised form of the force-indentation models are presented. Section 4 discusses the results from the simulation of the present model in comparison with experimental data and results from other models. Finally, section 5 gives the conclusions of the present study.

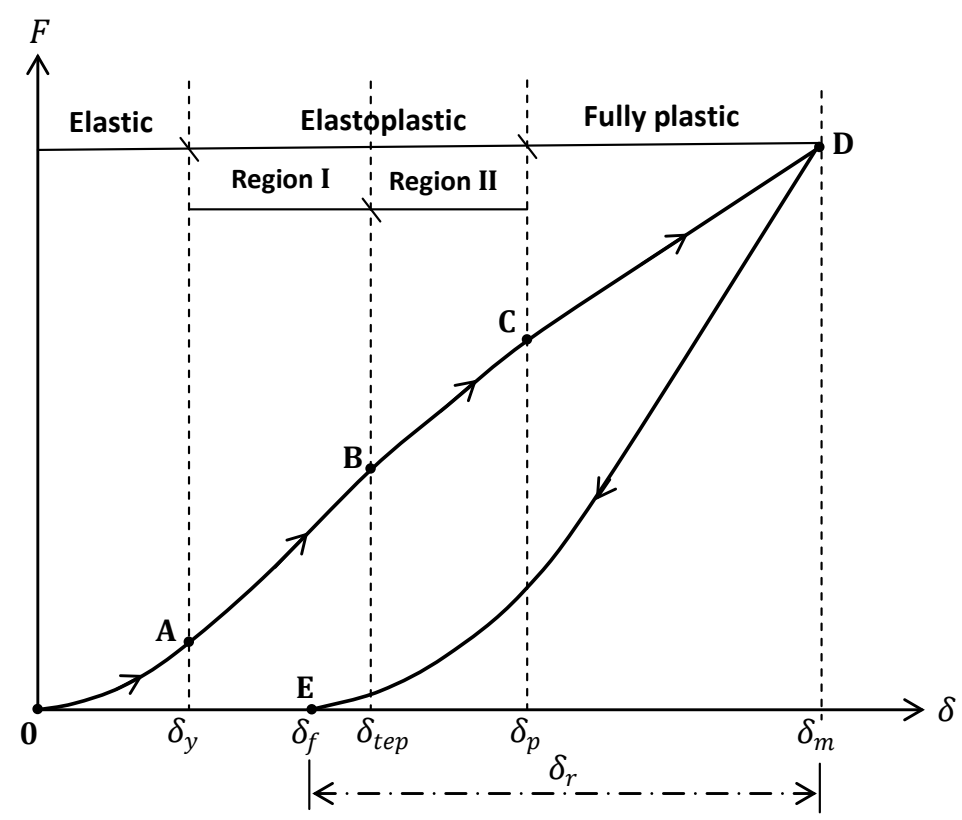

Figure 2: Sketch of compliance curve for the new contact law 


\section{Model development}

Static contact models can be used for modelling the impact response of structures by redefining the local indentation during impact as the relative displacement between the impactor and the target. The equation of motion of an impactor with an initial velocity of $V_{0}$ when in contact with a half space target is given as:

$$
m \ddot{\delta}+F=0
$$

where $m$ is the mass of the impactor; $\delta$ is the indentation, which is equal to the displacement of the impactor for half-space analysis, and the initial conditions are $\delta(0)=0$, $\dot{\delta}(0)=V_{0} ; F$ is the impact force and it is subject to the restriction in equation (2). The impact force, $F$, is related to the indentation and can be determined from an appropriate contact model. It is the primary objective of this paper to develop a simple and reasonably accurate contact model that accounts for plastic deformation effects during post-yield loading and unloading, and can be easily applied to impact modelling. This does not exclude the application of the present model for validation of indentation test results and finite element models.

$$
F=\left\{\begin{array}{cl}
F(\delta) & \text { if } F>0 \\
0 & \text { if } F \leq 0
\end{array}\right.
$$

\subsection{Force-indentation relationships}

During contact loading, the elastoplastic loading regime is initiated immediately after the target material yields. As a result of yielding, a plastically-deforming material appears below the contact surface at a depth of about half the contact radius (Figure 3a). This plastically-deforming material is completely enclosed within a surrounding elasticallydeforming material [1]. As the elastoplastic loading progresses, the plastically-deforming material increases and the surrounding elastically-deforming material decreases. Eventually, perfectly plastic conditions are met when the plastically-deforming material breaks free to the contact surface and comes in contact with the indenter. This marks the end of the elastoplastic loading regime and the onset of the fully plastic loading regime.

Previous contact models $[1,5,6,8]$ that are based on the three loading regimes have used single continuous functions to model the elastoplastic loading regime. However, experimental evidence and FEA results reveal that there is a linear loading region preceding the onset of fully plastic loading predicted by indentation theory [7]. Also, indentation theory and experimental evidence reveal that a nonlinear loading exists in the elastoplastic regime [7]. Therefore, it is proposed here that the elastoplastic loading regime can be divided into two regions as follows:

a. Region I: this is characterised by a nonlinear force-indentation relationship. The onset of this region is the yield point and it ends somewhere in the elastoplastic regime. In this region, the plastically-deforming material is well below the contact surface and it is smaller in comparison to the surrounding elastically-deforming material (Figure $3 b$ ). This means that the plastically-deforming material is fully contained within the 
surrounding elastic material and the indenter is only in contact with the elasticallydeforming material.

b. Region II: this is characterised by a linear force-indentation relationship. The region commences from the terminus of region I and ends at the onset of fully plastic conditions. Here, the plastically-deforming material is considered to be comparable or larger than the surrounding elastically-deforming material (Figure $3 \mathrm{c}$ ). The plastically deforming material grows closer to the contact surface, and breaks free to the contact surface at the end of this region.

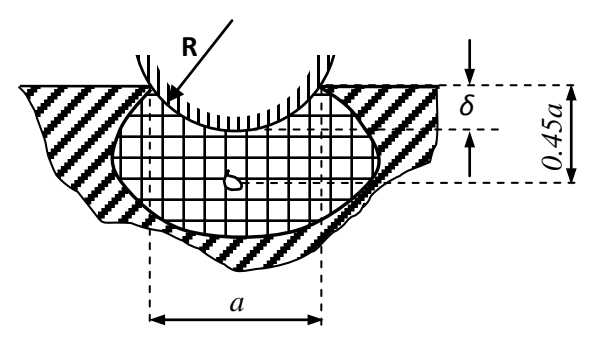

(a)

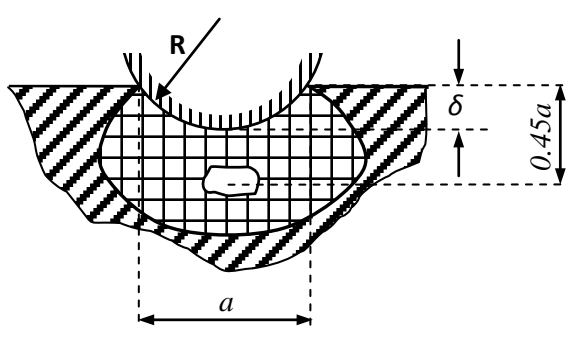

(b)

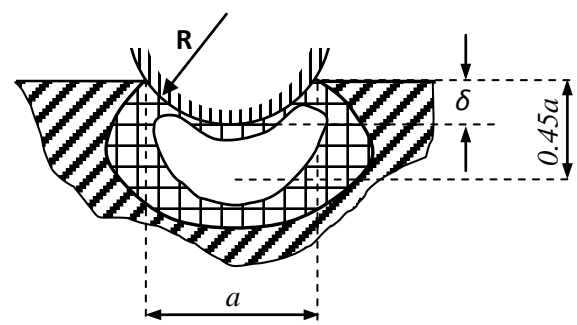

(c)

Figure 3: Plastic deformation in the elastoplastic loading regime (a) at yield (b) during nonlinear elastoplastic deformation (c) during linear elastoplastic deformation. The cross-hatched area is the surrounding elastically-deforming material while the unshaded area is the plastically-deforming material.

In the present study, it is proposed that region I can be modelled by a modified form of the Hertz contact law that incorporates a yield effect. This region is modelled in this way since it has similar characteristics as the elastic loading stage namely: (i) it is nonlinear, and (ii) its deformation can be considered to be effectively elastic-like since the plastically-deforming material is small and fully contained within the surrounding elastically-deforming material. Region II is modelled using a linear force-indentation that incorporates the appropriate plastic deformation effect, which is assumed to occur at transition point in the elastoplastic loading regime.

The features of these two regions can be seen in the approximate analytical models for the elastoplastic loading regime presented in $[1,5]$. These models incorporate plastic deformation effect and the nonlinear part of the elastoplastic loading appears as a logarithmic function of the indentation. The existence of the two regions in the elastoplastic 
loading regimes means that there is a transition point from region I to region II. Majeed and co-workers [7], following observations of FEA results, assumed that the transition from region I to region II occurs at the point where the mean contact pressure is equal to the arithmetic average of the mean contact pressures at yield and at fully plastic conditions i.e. the transition occurs at $P_{0}=1.95 S_{y}$. Whereas this assumption produced results that are in good agreement with experimental data for the case study they investigated, it requires further validation. Here the indentation and mean contact pressure at the transition point are derived analytically from continuity conditions and existing indentation theory.

Based on the foregoing discussions, the following force-indentation relationships are proposed for modelling the loading and unloading during elastoplastic indentation.

\section{Elastic loading regime}

$$
F_{e}=K_{h} \delta^{3 / 2} \quad 0 \leq \delta \leq \delta_{y}
$$

\section{Elastoplastic loading regime}

- Region I: Nonlinear elastoplastic loading

$$
F_{e p}^{I}=K_{h}\left(\delta-\delta_{y}\right)^{3 / 2}+K_{h} \delta_{y}^{3 / 2} \quad \delta_{y} \leq \delta \leq \delta_{t e p}
$$

- Region II: Linear elastoplastic loading

$$
F_{e p}^{I I}=K_{l}\left(\delta-\delta_{\text {tep }}\right)+K_{h}\left[\left(\delta_{\text {tep }}-\delta_{y}\right)^{3 / 2}+\delta_{y}^{3 / 2}\right] \quad \delta_{\text {tep }} \leq \delta \leq \delta_{p}
$$

Fully plastic loading regime

$$
F_{f p}=K_{p}\left(\delta-\delta_{p}\right)+F_{\delta=\delta_{p}} \quad \delta_{p} \leq \delta \leq \delta_{m}
$$

Restitution (unloading)

$$
F_{u}=K_{u}\left(\delta-\delta_{f}\right)^{3 / 2} \quad \delta_{f} \leq \delta \leq \delta_{m}
$$

where $F_{e}, F_{e p}, F_{f p}$, and $F_{u}$ are the elastic, elastoplastic, fully plastic, and unloading forces respectively. The superscripts on $F_{e p}$ indicate the region in the elastoplastic regime, and $F_{\delta=\delta_{p}}$ is the value of the contact force at the onset of fully plastic conditions. $K_{h}$ is the Hertzian contact stiffness, $K_{l}$ is the linear stiffness in region II, $K_{p}$ is the linear stiffness during the fully plastic regime, and $K_{u}$ is the nonlinear stiffness during unloading. $\delta_{y}$ is the indentation at yield, $\delta_{\text {tep }}$ is the indentation at the transition between region I and region II, $\delta_{p}$ is the indentation at the onset of fully plastic conditions, and $\delta_{f}$ is the fixed or permanent or non-recoverable indentation. The constants in the model are derived in the next subsection.

\subsection{Determination of constants in the contact model}

The constants in the force-indention relationships, equations (3) - (7), are derived from indentation theory and continuity conditions as follows.

\section{Elastic loading regime}

The only constant in this regime is $K_{h}$ and it is determined from the Hertz contact law.

$$
K_{h}=\frac{4}{3} E R^{1 / 2}
$$


where $E$ and $R$ are the effective modulus and radius respectively, and they are given by: $R=\left[1 / R_{i}+1 / R_{t}\right]^{-1}$ and $E=\left[\left(1-v_{i}^{2}\right) / E_{i}+\left(1-v_{t}^{2}\right) / E_{t}\right]^{-1}$. The subscripts $i$ and $t$ stand for impactor and target respectively, and $v$ is the Poisson's ratio.

\section{Region I: Nonlinear elastoplastic loading}

Majeed et al [7] modelled this region using Hertz's law and therefore, the effect of post-yield deformation was not accounted for. These have now been accounted for in the present model as shown in equation (4). The unknown constants in this region are $K_{h}$ and $\delta_{y}$, and $K_{h}$ is determined from equation (8). To derive the expression for $\delta_{y}$ the elastic force at $\delta=\delta_{y}$ is equated to the product of the mean pressure and contact area at $\delta=\delta_{y}$. Hence,

$$
\left(F_{e}\right)_{\delta=\delta_{y}}=F_{\delta=\delta_{y}}
$$

where $F_{\delta=\delta_{y}}$ is the contact force at yield expressed in terms of the mean pressure and following [9], can be written as $F_{\delta=\delta_{y}}=\pi R P_{0 y} \delta_{y} ; P_{0 y}=1.1 S_{y}$ is the mean pressure at yield. $\left(F_{e}\right)_{\delta=\delta_{y}}$ is evaluated by substituting $\delta=\delta_{y}$ in equation (3). Therefore,

$$
K_{h} \delta_{y}^{3 / 2}=1.1 \pi R S_{y} \delta_{y}
$$

from which the expression for $\delta_{y}$ is obtained as:

$$
\delta_{y}=\left(\frac{1.1 \pi R S_{y}}{K_{h}}\right)^{2}
$$

Substituting equation (8) into equation (9),

$$
\delta_{y}=\frac{0.681 \pi^{2} R S_{y}^{2}}{E^{2}}
$$

\section{Fully plastic loading regime}

For convenience, expressions for the constants of the fully plastic loading regime are derived before those of the second region of the elastoplastic loading regime. The unknown constants here are $K_{p}, \delta_{p}$ and $F_{\delta=\delta_{p}}$. As mentioned earlier, the mean pressure in this regime is constant and therefore, the contact force at any point in this regime can be written as:

$$
F_{f p}=\pi P_{0 p} a^{2}
$$

where $P_{0 p}=2.8 S_{y}$ is the mean contact pressure at fully plastic conditions, and $a$ is the contact radius. Since the force-indentation relationship in this loading regime is linear, $a^{2} \propto \delta$. A general relationship between the contact radius and indentation has been proposed as $a^{2}=2 R \delta+c$ [8], where the constant $c=a_{p}^{2}-2 R \delta_{p} \neq 0$ can be either positive of negative depending on the values of $a_{p}$ and $\delta_{p}$. Therefore,

$$
a^{2}=2 R\left(\delta-\delta_{p}\right)+a_{p}^{2}
$$

Substituting equation (12) in equation (11) and comparing the resulting expression with equation (6), it can be deduced that

$$
K_{p}=5.6 \pi R S_{y}
$$

and 


$$
F_{\delta=\delta_{p}}=\pi P_{0 p} a_{p}^{2}=2.8 \pi S_{y} a_{p}^{2}
$$

where $a_{p}$ can be calculated according to [5] as: $a_{p}^{2}=R\left(2 \delta_{p}-\delta_{y}\right)$. When equation (13) was used for the simulation of the force-indentation curve of selected materials, the impact force predictions obtained during the fully loading regime were significantly higher than the experimental data $[10,11]$ to which the predictions were compared. This is because equation (13) is for the case of a completely rigid indenter, but the indenters used in the experiments are not completely rigid. Brake [8] accounted for the effect of this relative rigidity of the contacting bodies by defining an effective hardness, which was used instead of the constant mean pressure at yield. Here the effect of the relative rigidity of the contacting bodies is accounted for by defining a correction factor as $\eta=H /\left(2.8 S_{y}\right)$; where $H$ is the effective hardness defined as [8]: $H=\left[1 / H_{i}+2 / H_{t}\right]^{-1}$. Based on the data of material properties provided by Brake [8] the correction factor was calculated as $\eta=0.81$ and $\eta=0.85$ for the experiments by Alcalá et al [10] and Bartier et al [11] respectively. A correction factor of 0.821 , which is close to the average correction factor for both experiments, was introduced in equation (13) and found to give good predictions of the experiments. Consequently, the constant $K_{p}$ is calculated as:

$$
K_{p}=4.6 \pi R S_{y}
$$

Note that the value of $K_{p}$ does not affect the elastoplastic response; but it determines the fully plastic response.

To evaluate completely the contact force in the fully plastic loading regime, the value or expression for $\delta_{p}$ or $\delta_{p} / \delta_{y}$ must be known. In the literature, different expressions have been used for the ratio $\delta_{p} / \delta_{y}$. Brake [8] used the expression $\delta_{p} / \delta_{y}=\left(P_{o p} / S_{y}\right)^{2}=7.84$ where $P_{o p}=2.8 S_{y}$. This expression is the von Mises equivalent of the expression given by Stronge [9] that is based on Tresca's yield criterion. It was observed that the use of this expression sometimes produces an unrealistic bend in the elastoplastic regime and a smoothing condition was applied to correct this. The condition states that the slope of the contact force at $\delta=\delta_{p}$ should be equal to the slope of the extended Hertzian force at the same indentation i.e. $\left(d F_{e} / d \delta\right)_{\delta=\delta_{p}}=\left(d F_{f p} / d \delta\right)_{\delta=\delta_{p}}$. Using equations (3) and (11), and $\delta_{y}=\left(\pi R P_{o y} / K_{h}\right)^{2}$ in the smoothing condition, the resulting ratio is $\delta_{p} / \delta_{y}=\left(4 P_{o p} /\right.$ $\left.3 P_{o y}\right)^{2}=13.94$ where $P_{o y}=S_{y}$ has been used in line with the expression for the unsmoothed case. In [5], an analytical formula for the elastoplastic loading regime that expresses the normalised mean pressure as a function of the normalised indentation was derived. Evaluating this formula at $\delta=\delta_{p}$ results in $\delta_{p} / \delta_{y}=82.5$, which translates into a force ratio of $F_{p} / F_{y}=417.5$. Johnson [1] gave a force ratio of $F_{p} / F_{y} \approx 400$, and based on his analytical formula for the fully plastic loading regime the ratio $\delta_{p} / \delta_{y} \approx 73$ can be obtained. Stronge [5] also reported a ratio of $\delta_{p} / \delta_{y} \approx 140$ and a corresponding force ratio of $F_{p} / F_{y} \approx 650$ from finite element studies of elastic-perfectly plastic bodies. The results of [1] and [5] are close and are based on theoretically valid analytical approximations of the elastoplastic loading regime. Therefore, a ratio of $\delta_{p} / \delta_{y}=82.5$ has been used in the present study. However, parametric studies based on the formulation of the elastoplastic 
regime of the current model reveal that $\delta_{p} / \delta_{y}$ can be reasonably varied from 28 to 140 as shown in later discussions in this paper. Hence,

$$
\delta_{p}=82.5 \delta_{y}=\frac{56.18 \pi^{2} R S_{y}^{2}}{E^{2}}
$$

\section{Region II: Linear elastoplastic loading}

In this region, two constants are to be determined; $K_{l}$ and $\delta_{\text {tep }}$. To obtain expressions for these constants, the following continuity conditions are applied.

Condition 1: The slope of region II is equal to the slope of region I at $\delta=\delta_{\text {tep }}$. The condition implies that the linear stiffness of region II is equal to the slope of region I at $\delta=\delta_{\text {tep }}$. This condition has been used [4, 7] when considering transition from a nonlinear behaviour to a linear behaviour. It is dimensionally consistent, and therefore has also been applied in the present modelling. The condition can be expressed mathematically as:

$$
\left(\frac{d F_{e p}^{I I}}{d \delta}\right)_{\delta=\delta_{t e p}}=\left(\frac{d F_{e p}^{I}}{d \delta}\right)_{\delta=\delta_{t e p}}
$$

from which we get:

$$
K_{l}=1.5 K_{h}\left(\delta_{\text {tep }}-\delta_{y}\right)^{1 / 2}
$$

Condition 2: The value of the linear elastoplastic force is equal to the value of the fully plastic force at $\delta=\delta_{p}$.

$$
\left(F_{e p}^{I I}\right)_{\delta=\delta_{p}}=\left(F_{f p}\right)_{\delta=\delta_{p}}
$$

Substituting equation (5) into this condition,

$$
K_{l}\left(\delta_{p}-\delta_{t e p}\right)+K_{h}\left[\left(\delta_{\text {tep }}-\delta_{y}\right)^{3 / 2}+\delta_{y}^{3 / 2}\right]=F_{\delta=\delta_{p}}
$$

From equation (18), $K_{l}$ can be expressed as:

$$
K_{l}=\frac{F_{\delta=\delta_{p}}-K_{h}\left[\left(\delta_{t e p}-\delta_{y}\right)^{3 / 2}+\delta_{y}^{3 / 2}\right]}{\left(\delta_{p}-\delta_{t e p}\right)}
$$

Using equations (17) and (19), the following nonlinear equation can be obtained.

$$
K_{h}\left(\delta_{t e p}-\delta_{y}\right)^{3 / 2}-1.5 K_{h}\left(\delta_{t e p}-\delta_{y}\right)^{1 / 2}\left(\delta_{t e p}-\delta_{p}\right)=F_{\delta=\delta_{p}}-K_{h} \delta_{y}^{3 / 2}
$$

Squaring both sides of equation (20) and after some algebraic simplifications (see appendix A), a cubic equation in $\delta_{\text {tep }}$ is obtained as shown in equation (21).

$$
\delta_{\text {tep }}^{3}-\Lambda_{1} \delta_{\text {tep }}^{2}+\Lambda_{2} \delta_{\text {tep }}-\Lambda_{3}=0
$$

where $\Lambda_{1}=6 \delta_{p}-3 \delta_{y} ; \Lambda_{2}=9 \delta_{p}^{2}-6 \delta_{y} \delta_{p} ; \Lambda_{3}=4 \delta_{y}^{3}-12 \delta_{y}^{2} \delta_{p}+9 \delta_{y} \delta_{p}^{2}+\left(2 Z / K_{h}\right)^{2}$; and $Z=F_{\delta=\delta_{p}}-K_{h} \delta_{y}^{3 / 2}$. The value of $\delta_{\text {tep }}$ obtained from equation (21) is substituted into equation (17) to calculate the value of $K_{l}$. The solution to equation (21) must be within the range $\delta_{y}<\delta_{\text {tep }}<\delta_{p}$. 


\section{Restitution (unloading)}

There are two unknown constants in this regime according to equation (7). The constants are the unloading stiffness $K_{u}$ and the non-recoverable indentation $\delta_{f}$. In order to determine the expressions for these constants, two new variables are needed as follows: the maximum indentation $\delta_{m}$ and the maximum contact force $F_{m}$. Since this regime is elastic, the force-indentation relationship is analogous to the Hertz contact model for elastic loading. For metallic targets, the constants can be determined according to [5] as follows:

$$
\begin{gathered}
K_{u}=\frac{4}{3} E R_{d}^{1 / 2} \\
\delta_{f}=\delta_{m}-\left(\frac{3 F_{m}}{4 E R_{d}^{1 / 2}}\right)^{2 / 3}
\end{gathered}
$$

where $R_{d}$ is the deformed effective radius, and $R_{d} \geq R$. Whereas the Hertz stiffness is related to the effective radius, the unloading stiffness is related to a deformed effective radius due to plastic deformation effects [5]. The essential difference between the present restitution model and that in [5] is in the way $R_{d}$ is determined. Following observations of previous experiments, Johnson [1] suggested that the change in the effective radius during elastoplastic loading can be neglected. He also observed that experimental investigations revealed a small significant change in the effective contact radius during fully plastic loading. Based on these observations, $R_{d}$ is calculated here as $R_{d} \approx R$ when the maximum penetration is in the elastoplastic regime or the initial phase of the fully plastic regime; and $R_{d} \approx R+\delta_{m} / 2$ when the maximum penetration is well into the fully plastic regime [8] e.g. $\delta_{m} / \delta_{p} \geq 2$. When the unloading starts from the elastic loading regime, $\delta_{f}=0$ and $R_{d}=R$.

\subsection{Determination of the maximum contact force and indentation of a half-space target from impact conditions}

Generally speaking, the relative velocity of the impactor and target during impact is zero at the maximum indentation. This condition can be used to determine the maximum indentation and contact force of a half-space impact that can be modelled with quasi-static assumptions by using energy balance principles. However, with impacts on slender (flexible) targets, this condition can occur more than once during the impact duration thereby resulting in more than one extremum. Furthermore, the interactions between vibrations of the target and the indentation introduce complications which make the energy balance approach impractical for impact on slender targets [3].

To define $K_{u}$ and $\delta_{f}$ completely, the maximum contact force and indentation must be known. To the best of the authors' knowledge, there is no comprehensive method in the literature for determining the maximum contact force and indentation of an elastoplastic half-space from the impact conditions. During impact on an elastoplastic half-space, the impact energy of the impactor is dissipated through various mechanisms such as elastic compression, elastic waves, plastic deformation, heat, sound, etc. Johnson [1] showed that for impact velocities up to $500 \mathrm{~m} / \mathrm{s}$, all other mechanisms of impact energy dissipation can 
be neglected except for elastic compression and plastic deformation. The elastic compression energy is restored back to the impactor during unloading and the plastic deformation is dissipated in the half-space target as a permanent indentation. The range of impact velocities up to $500 \mathrm{~m} / \mathrm{s}$ covers low to medium velocity impacts where strain rate effects can be neglected, and therefore, the impact event can be modelled using quasi-static assumptions [1]. For such impact events, the energy balance approach can be applied with the following assumptions: (i) that no energy is lost from the impact system, and (ii) that the velocity of the impactor is momentarily zero at maximum indentation, at which point the kinetic energy of the impactor would have been completely used for deformation of the target i.e. elastic compression and plastic deformation. Based on the energy balance approach, the following expression holds for low to medium velocity impact:

$$
\frac{1}{2} m V_{0}=\int_{0}^{\delta_{m}} F d \delta
$$

where $F$ represents a general contact force that can be in any of the loading regimes.

In a typical impact event, a spherical impactor with known mass and velocity impacting a half-space target produces a definite maximum indentation that falls into one of the loading regimes discussed above. Therefore, it is necessary to determine the loading regime in which the maximum indentation is located, so that the appropriate forceindentation relationship(s) can be used in the impact analysis. Since the contact force has been represented as a piecewise continuous function of indentation, an iterative algorithm based on the energy balance is proposed here to determine the maximum indentation and consequently the maximum contact force. In order to use this method knowledge of all the constants in the loading regimes is required. In view of the fact that the contact loading starts from the elastic regime, the first step is to compare the total deformation work done in the elastic regime $\left(W_{y}=W_{e}\right)$ to the kinetic energy $(K E)$ of the impactor. If $K E<W_{y}$, then the maximum indentation is in the elastic region, otherwise we proceed to determine the total deformation work done up the end of region I ( $\left.W_{t e p}\right)$ and compare this with $K E$. Again, if $K E<W_{\text {tep }}$, then the maximum indentation is in region $\mathrm{I}$, otherwise the process is repeated until the maximum indentation is located in one of the loading regimes. The algorithm for this procedure is shown in the flowchart in Figure 4. Also, the total deformation work done from the beginning of the loading to each of the transition points in the loading regime are derived in appendix $B$. 


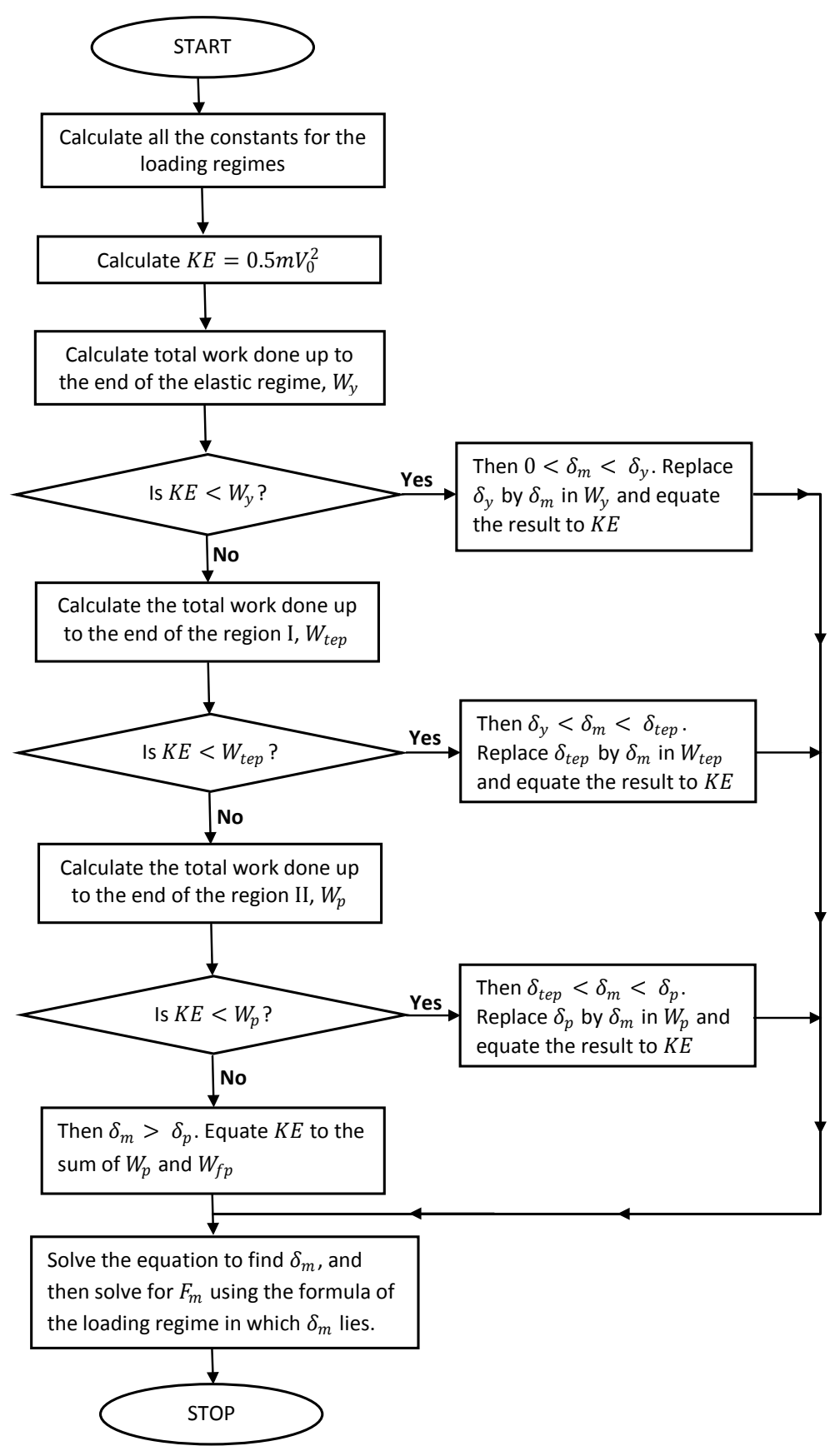

Figure 4: Method for determining the maximum indentation and contact force during impact.

\section{Normalised form of the contact model}

In this section, the contact model presented in equations (3) to (7) is normalised with respect to the contact force at yield. The normalised contact model is more elegant and easier to compute. Dividing equations (3) to (7) by $F_{y}=K_{h} \delta_{y}^{3 / 2}$, the normalised form of the present contact model is derived as shown.

\section{Elastic loading regime}

$$
\bar{F}_{e}=\bar{\delta}^{3 / 2} \quad 0 \leq \bar{\delta} \leq 1
$$




\section{Elastoplastic loading regime}

- $\quad$ Region I: Nonlinear elastoplastic loading

$$
\bar{F}_{e p}^{I}=(\bar{\delta}-1)^{3 / 2}+1 \quad 1 \leq \bar{\delta} \leq \bar{\delta}_{t e p}
$$

- Region II: Linear elastoplastic loading

$$
\bar{F}_{e p}^{I I}=1.5\left(\bar{\delta}_{t e p}-1\right)^{1 / 2}\left(\bar{\delta}-\bar{\delta}_{t e p}\right)+\left(\bar{\delta}_{t e p}-1\right)^{3 / 2}+1 \quad \bar{\delta}_{t e p} \leq \bar{\delta} \leq \bar{\delta}_{p}
$$

Fully plastic loading regime

$$
\bar{F}_{f p}=4.182\left(\bar{\delta}-\bar{\delta}_{p}\right)+2.545\left(2 \bar{\delta}_{p}-1\right) \quad \bar{\delta}_{p} \leq \bar{\delta} \leq \bar{\delta}_{m}
$$

Restitution (unloading)

$$
\bar{F}_{u}=\bar{K}\left(\bar{\delta}-\bar{\delta}_{f}\right)^{3 / 2} \quad \bar{\delta}_{f} \leq \bar{\delta} \leq \bar{\delta}_{m}
$$

In equations (26) to (29), the bar over the forces indicate normalisation with respect to $F_{y}$ and the bar over the indentations indicate normalisation with respect to $\delta_{y}$ e.g. $\bar{F}_{e}=F_{e} / F_{y}$ and $\bar{\delta}=\delta / \delta_{y}$. The normalised constants are given as: $\bar{\delta}_{\text {tep }}$ lies within the range $1<\bar{\delta}_{\text {tep }}<$ $\bar{\delta}_{p}$, and it is obtained from the equation

$$
\bar{\delta}_{\text {tep }}^{3}-\Omega_{1} \bar{\delta}_{\text {tep }}^{2}+\Omega_{2} \bar{\delta}_{t e p}-\Omega_{3}=0
$$

where $\Omega_{1}=6 \bar{\delta}_{p}-3 ; \quad \Omega_{2}=9 \bar{\delta}_{p}^{2}-6 \bar{\delta}_{p} ;$ and $\Omega_{3}=54.268-156.352 \bar{\delta}_{p}+112.632 \bar{\delta}_{p}^{2}$. Also, $\bar{\delta}_{p}=82.5 ; \bar{K}=R_{d} / R$ and $\bar{\delta}_{f}=\bar{\delta}_{m}-\left(3 F_{m} / 4 E R_{d}^{1 / 2}\right)^{2 / 3} / \delta_{y}$. Equation (30) can be obtained by dividing equation (21) by $\delta_{y}^{3}$ or by solving equations (27) and (28) simultaneously at $\bar{\delta}=\bar{\delta}_{p}$ using the same approach discussed in appendix A. Given that all the coefficients of the cubic equation in $\bar{\delta}_{t e p}$ are dependent on $\bar{\delta}_{p}$, then substituting $\bar{\delta}_{p}=82.5$ and solving equation (30) gives $\bar{\delta}_{\text {tep }}=13.93$.

\section{Results and discussion}

\subsection{Some observations on the present contact model}

To understand the features and workings of the present contact model and to compare with findings of published analytical models and finite element analysis, parametric studies were carried out on the present model.

Using Brake's contact model [8], it was shown that the smoothed formulation for the elastoplastic loading regime always produced the best result and $\bar{\delta}_{p}=13.94$ according to the smoothed formulation. In the current model, the transition point in the elastoplastic loading regime was found to occur at $\bar{\delta}_{t e p}=13.93$. This point coincides with the point at which Brake's smoothed formulation assumes a transition to the fully plastic regime. The implication is that a transition from nonlinear to linear deformation behaviour occurs at this point. Whereas Brake's model proposed that this point (i.e. $\bar{\delta} \cong 13.93$ ) marks the onset of fully plastic conditions, the present model indicates that the point is located within the elastoplastic loading regime and gives rise to a linear elastoplastic deformation just before fully plastic conditions are reached. The presence of linear elastoplastic deformation 
behaviour during contact loading has been reported [7] and can also be seen in the compliance curve for elastoplastic indentation presented in [1].

When $\bar{\delta}_{t e p}=13.93$ is substituted into equation (27), the normalised force at the transition point in the elastoplastic loading regime is calculated to be $\bar{F}_{\text {tep }}=47.5$. According to [5], the normalised contact force in the elastoplastic loading regime can be expressed in terms of the normalised mean pressure and indentation as: $F / F_{y}=$ $\left(P_{0} / P_{0 y}\right)\left(2 \delta / \delta_{y}-1\right)$. Substituting $\bar{\delta}_{t e p}=13.93, \bar{F}_{t e p}=47.5$, and noting that $P_{o y}=1.1 S_{y}$, the mean pressure at $\bar{\delta}=\bar{\delta}_{\text {tep }}$ was calculated to be equal to $1.945 S_{y}$. This value, which is approximately equal to $1.95 S_{y}$, is in agreement with the transition mean pressure in the elastoplastic loading regime as proposed by Majeed and co-workers [7]. However, according to the model in [7] the transition mean pressure occurs when $\bar{\delta} \cong 3.15$, whereas for the present model it occurs at $\bar{\delta}=13.93$. In agreement with the Brake's model [8] the present contact model confirms that the contact deformation changes from nonlinear to linear behaviour when the normalised indentation is equal to 13.94; and in agreement with the model of Majeed and co-workers [7] the present contact model confirms that the contact deformation in the elastoplastic loading regime changes from nonlinear to linear behaviour at a mean pressure of $1.95 S_{y}$. Since the non-dimensional indentation of 13.94 and the mean pressure of $1.95 S_{y}$ occur at the same point according to the present model, it appears that these values are unique constants for the transition point in the elastoplastic loading regime.

The transition point in the elastoplastic loading regime of the current model lies in the range $1<\bar{\delta}_{t e p}<\bar{\delta}_{p}$, and this transition point is determined from the cubic equation that was derived from continuity conditions. In a limiting case, $\bar{\delta}_{\text {tep }}$ will be equal to $\bar{\delta}_{p}$, but it is not possible for $\bar{\delta}_{t e p}$ to be greater than $\bar{\delta}_{p}$. Using these practical restrictions, the possible range of applicable values for $\bar{\delta}_{\text {tep }}$ was investigated. It was observed that only one of the roots of the cubic equation satisfied the restrictions, and an increase in the value of $\bar{\delta}_{p}$ resulted in a decrease in the calculated value of $\bar{\delta}_{t e p}$ and vice versa. It was found that $\bar{\delta}_{\text {tep }}=\bar{\delta}_{p}$ when $\bar{\delta}_{p} \cong 27.5$, which is the lower bound for $\bar{\delta}_{p}$ based on the present model. Although the lower bound for $\bar{\delta}_{p}$ is much lower than the values proposed by Johnson [1] and Stronge [5], values of $\bar{\delta}_{p}$ less than 10 have been used is some models [8] and shown to give good predictions of the fully plastic loading regime when compared with static indentation experiments. Since values of $\bar{\delta}_{p}$ up to 140 have been reported [5], the present model suggests that $\bar{\delta}_{p}$ can be logically varied within the range of $28 \leq \bar{\delta}_{p} \leq 140$. The corresponding range for $\bar{\delta}_{\text {tep }}$ calculated from equation (30) is $23.84 \geq \bar{\delta}_{\text {tep }} \geq 13.28$. Note that the value of $\bar{\delta}_{\text {tep }}$ does not change much between $\bar{\delta}_{p}=82.5$ (where $\bar{\delta}_{\text {tep }}=13.93$ ) and $\bar{\delta}_{p}=140$ (where $\bar{\delta}_{\text {tep }}=13.28$ ).

Finite elements analysis $[5,6]$ and analytical studies $[1,5,8]$ have used different values for the normalised indentation at the onset of fully plastic loading. It is proposed here that a value of $\bar{\delta}_{p}=82.5$ with a corresponding value of $\bar{\delta}_{t e p}=13.93$ can be used for 
modelling a static or dynamic elastoplastic indentation based on the present model. However, for cases where using these values in the present model results in predictions that deviate from what is expected e.g. experimental data, $\bar{\delta}_{p}$ can be varied within the range of $28 \leq \bar{\delta}_{p} \leq 140$ and the corresponding value of $\bar{\delta}_{\text {tep }}$ can be obtained by solving equation (30).

\subsection{Static indentation analysis}

To validate the present contact model, static indentation compliance curves obtained from the simulation of the present contact model was compared with experimental results $[10,11]$ and the model of Brake [8]. Brake's model was used for comparison with the present model because it was compared with nine other published models and shown to perform better in predicting the experimental results considered in his study [8]. Furthermore, his model can be evaluated completely from the contact geometry and material properties of the contact system just like the present contact model.

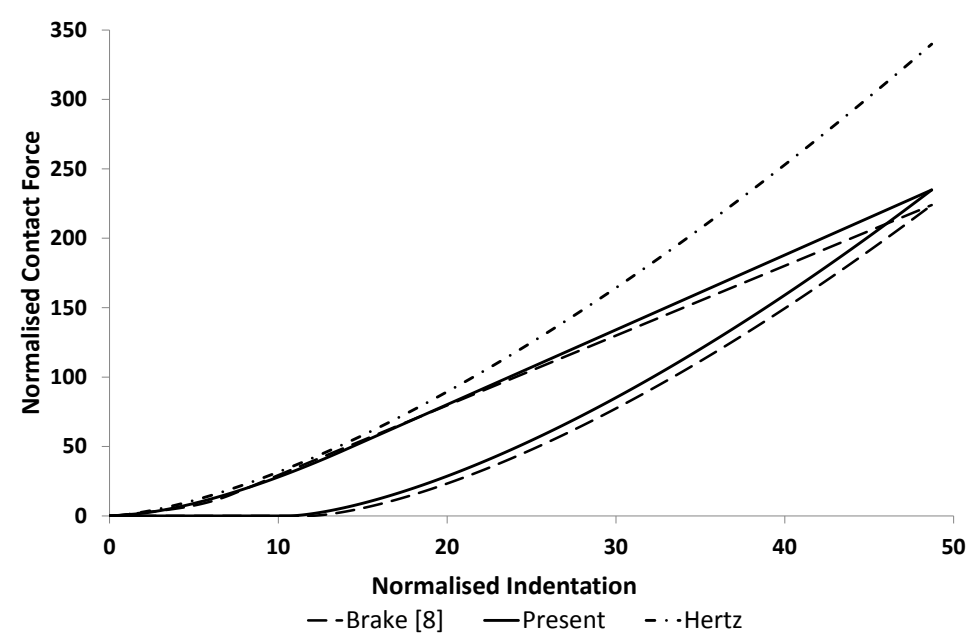

Figure 5: Compliance curve showing the Hertz contact model and the present model for indentation of two identical SUJ2 Steel balls.

In Figure 5 the compliance curve for the impact between two identical steel balls obtained using the present contact model is shown. The same problem was studied in [8] and the model results produced an unrealistic bend at the beginning of the elastoplastic loading regime (see exploded plot in Figure 5). Brake [8] corrected this bend using a smoothing condition. The present contact model shows no such bend and gives a maximum normalised contact force of 234 at a maximum normalised indentation of 48.7 as compared to 224 from the simulation of Brake's model. Also, the maximum conditions are located in the linear elastoplastic loading regime of the present model, whereas they are located in the fully plastic regime in [8]. However, the loading and unloading paths predicted by both models are in agreement. 
Table 1: Material properties for indentation of pure Nickel by spherical 10\% Cobalt-Tungsten Carbide Indenter

\begin{tabular}{lcc}
\hline Material Property & Indenter (Tungsten Carbide) & Target (Pure Nickel) \\
\hline Density [kg/m ${ }^{3}$ ] & 14500 & 8880 \\
Elastic Modulus [GPa] & 475 & 207 \\
Poisson's ratio [ - ] & 0.22 & 0.31 \\
Yield Strength [MPa] & - & 148 \\
Hardness [MPa] & 11436.6 & 695.8 \\
Radius [m] & $7.95 \times 10^{-4}$ & $\infty$ \\
\hline
\end{tabular}

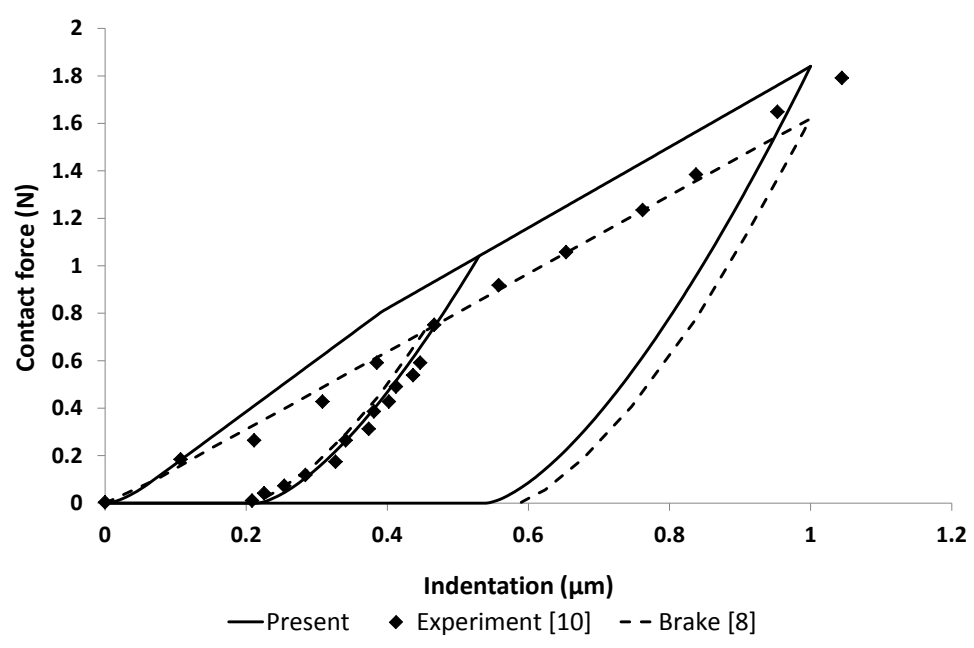

Figure 6: Comparison of compliance curve of present model with Brake [8], and experimental results [10] for indentation of pure Nickel. The maximum indentation is well into the fully plastic regime.

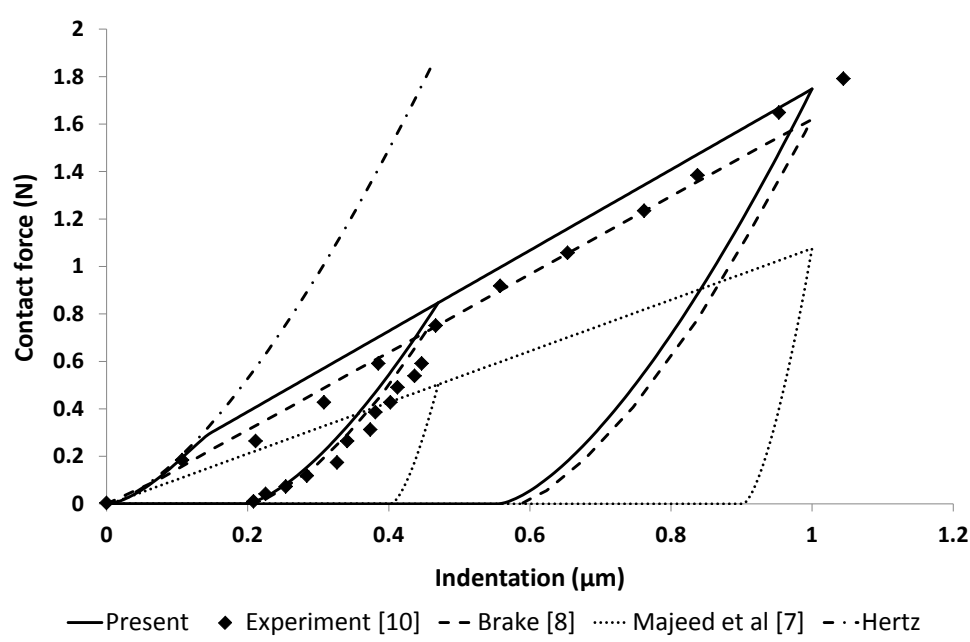

Figure 7: Comparison of compliance curve of present model with Brake [8], Majeed et al [7], and experimental results [10] for indentation of pure Nickel. The onset of fully plastic loading of the present model was adjusted for better fit of the experimental results.

Figure 6 illustrates the results of a tungsten carbide indenter on a pure nickel target. The properties of the contact system are given in Table 1 . A comparison is made between the results from present contact model, Brake's model [8], and measurements of Alcalá and 
co-workers [10] as reported in [8]. In Figure 6, the present model was simulated with $\bar{\delta}_{p}=82.5$ and a corresponding value of $\bar{\delta}_{t e p}=13.93$. The figure shows that the fully plastic loading predicted by the present models deviates significantly from the measured data. To obtain a better prediction of the fully plastic loading, $\bar{\delta}_{p}$ was assumed to be equal to 30 and the corresponding value for $\bar{\delta}_{\text {tep }}$ was calculated as 20.6. The compliance curve in this case is illustrated in Figure 7, which shows that the present model gives a good estimate of the measured data. The effect of setting $\bar{\delta}_{p}=30$ from the proposed general value of $\bar{\delta}_{p}=82.5$ is that the indentation range of region II is shortened i.e. $20.6<\bar{\delta}_{\text {tep }}<30$. Since shortening this region gives a better estimate of the measured data for the indentation of the pure nickel target, then the present model suggests that the pure nickel target undergoes more nonlinear elastoplastic deformation than linear elastoplastic deformation. The results from the model of Majeed and co-workers [7] and the Hertz contact model are also illustrated in Figure 7, and it can be seen that the loading path is underestimated by the model of Majeed and co-workers. While the contact model of Majeed and co-workers does not account for fully plastic loading, the inclusion of a fully plastic model would not improve the results because the contact stiffness in the fully plastic region is reduced further compared to the elastoplastic region, thereby leading to lower contact force estimates.

Table 2: Material properties for indentation of AISI 1035 Steel by spherical Tungsten Carbide Indenter

\begin{tabular}{lcc}
\hline Material Property & Indenter (Tungsten Carbide) & Target (AISI 1035 Steel) \\
\hline Density [kg/m ${ }^{3}$ ] & 14500 & 7850 \\
Elastic Modulus [GPa] & 600 & 210 \\
Poisson's ratio [ - ] & 0.28 & 0.30 \\
Yield Strength [MPa] & - & 300 \\
Hardness [MPa] & 11436.6 & 1528.8 \\
Radius [m] & $1.25 \times 10^{-3}$ & $\infty$ \\
\hline
\end{tabular}

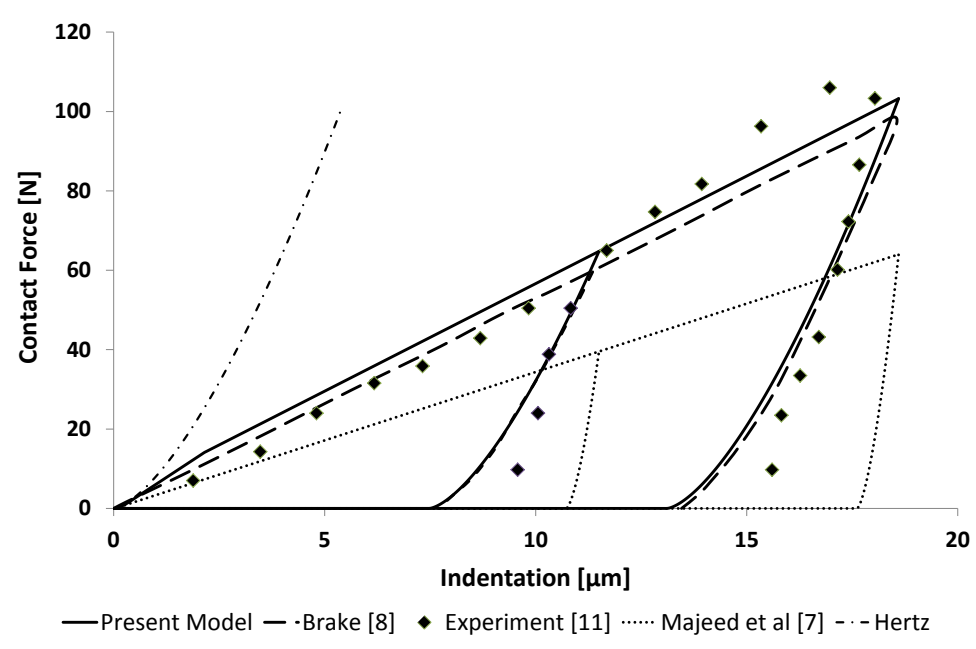

Figure 8: Comparison of compliance curve of present model with Brake [8], Majeed et al [7], and experimental results [11] for indentation of AISI 1035 Steel. The maximum indentation is well into the fully plastic regime. 
Another case considered was the indentation of an AISI 1035 steel target by a tungsten carbide indenter. The properties of the contact system are given in Table 2 and the compliance curve is shown in Figure 8 . The results of the present model were simulated for $\bar{\delta}_{p}=82.5$ and $\bar{\delta}_{t e p}=13.93$, and compared with the results from Brake's model [8], the model of Majeed and co-workers [7], and the measured data of Bartier and co-workers [11] as reported in [8]. The present model is shown to give a good prediction of the measured data. Although the unloading path of the present restitution model and that of Brake are in close agreement, both deviate from the experimental data towards the end of the unloading. When the maximum penetration is in the elastoplastic loading regime or in the early stages of the fully plastic loading, the effect of piling and sinking on the unloading response can be neglected [1]. When the maximum penetration is well into the fully plastic loading regime (as in this case), excessive unconstrained flow of the plastically-deformed material occurs and the effect of piling and sinking on the unloading may become significant. The deviation of the results of the present restitution model and that of Brake from the experimental data towards the end of the unloading can be attributed to the effect of piling and sinking, which is neglected by both restitution models. Also, the present model suggests that the steel target undergoes more linear elastoplastic deformation than nonlinear elastoplastic deformation. Again, the model of Majeed and co-workers underestimates the measured loading force and overestimates the permanent indentation at the end of the restitution.

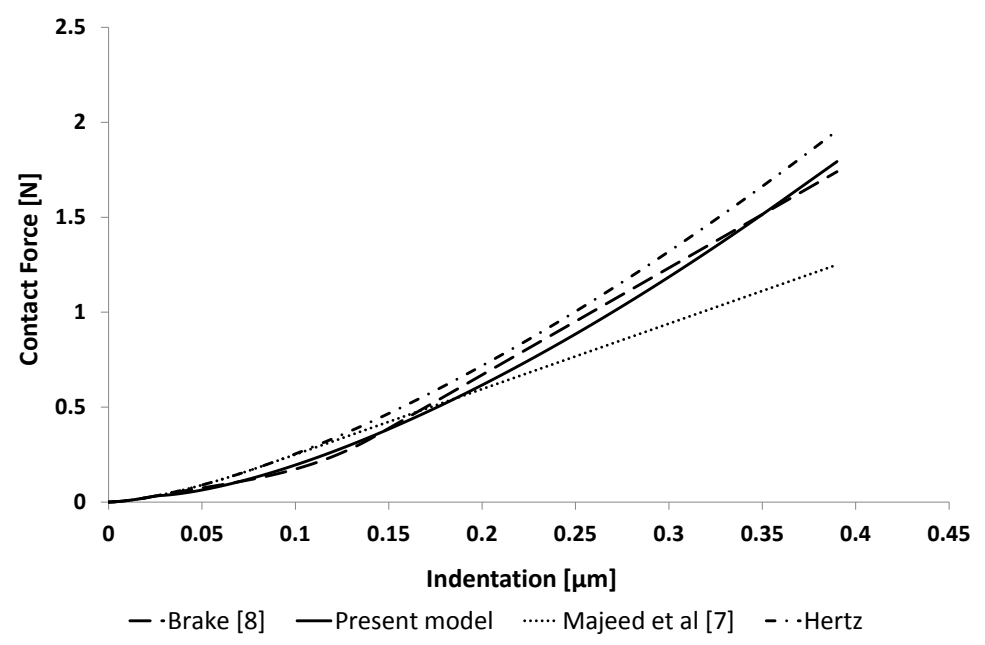

Figure 9: Comparison of elastoplastic loading of present model with the models of Brake [8], Majeed et al [7], and Hertz for indentation of AISI 1035 Steel.

For the metallic targets considered so far, the maximum indentation is well into the fully plastic regime and therefore, the curves in Figures 7 and 8 do not show the performance of the various models in the elastoplastic loading regime. In order to examine the performance of the various models in the elastoplastic region the indention of AISI 1035 steel target by a tungsten carbide indenter considered earlier was resimulated as shown in Figure 9. The maximum indention in this simulation is approximately 15 times the 
indentation at yield, and this has been chosen to reveal the elastoplastic regime of each model. The figure shows that the loading path of the present model and that of Brake are close, whereas the model of Majeed and co-workers begins to deviate when the indentation is about 7.5 times the indentation at yield, thus leading to an underestimation of the contact force beyond this point. This investigation is important because it confirms that the present model performs well in the elastoplastic regime of the loading phase. Hence, the combination of its simplicity, its ability to predict post-yield contact indentation with reasonable accuracy, and the fact that it can be completely evaluated from the properties of the contact system makes the present contact model an attractive choice for impact analysis and validation indentation test.

\section{Conclusions}

Contact models that account for plastic deformation during static indentation of a half-space have only evolved in the last half of the century. Most attempts by previous investigators have been based on elastic-perfectly plastic material behaviour but a few have developed models based on an elastic-elastoplastic-perfectly plastic material behaviour. In the present study a new contact model that accounts for plastic deformation in the loading and restitution phases of the contact force has been developed for half-space indentation by a spherical indenter, and it is based on elastic-elastoplastic-perfectly plastic material behaviour. In line with observations from previous experimental and FEA results reported in the literature [7], the elastoplastic loading regime was divided into two regions, nonlinear and linear, and modelled accordingly. Simple expressions have been derived for all constants in the present contact model and all the constants can be completely evaluated from the geometric and material properties of the contact system. Also, the model for the restitution phase allows for unloading from any of the loading regimes. The results of the study show that the present contact model works well in predicting the post-yield indentation of metallic targets.

Additionally, a comprehensive approach for calculating the maximum indentation and contact force of a half-space target from the impact conditions has been proposed in this study for the first time. The approach applies energy balance principles and it is applicable to impact events that can be modelled using quasi-static assumptions i.e. low to medium velocity impacts.

\section{Acknowledgements}

The first author wishes to acknowledge the Commonwealth Scholarship Commission (CSC), UK for funding his PhD research (CSC Award Ref.: NGCA-2011-60), of which this work is a part. 


\section{References}

[1]. Johnson, K.L. (1985): Contact Mechanics, Cambridge University Press, Cambridge.

[2]. Goldsmith, W. (2001): Impact: The Theory and Physical Behaviour of Colliding Solids, Dover Publications, New York.

[3]. Abrate, S. (1998): Impact on Composite Structures, Cambridge University Press, Cambridge.

[4]. Yigit, A.S. and Christoforou, A.P. (1994): On the Impact of a Spherical Indenter and an Elastic-Plastic Isotropic Half-Space, Composite Engineering, 4 (11), pp $1143-1152$.

[5]. Stronge, W.J. (2000a): Impact Mechanics, Cambridge University Press, Cambridge.

[6]. Li, L.Y., Wu, C.Y., Thornton, C. (2002): A theoretical model for the contact of elastoplastic bodies, Proc. IMechE, Part C: Journal of Mechanical Engineering Science, 216, pp $421-431$.

[7]. Majeed, M.A., Yigit, A.S., Christoforou, A.P. (2012): Elastoplastic Contact/Impact of Rigidly Supported Composites, Composites: Part B, 43, pp 1244-1251.

[8]. Brake, M.R. (2012): An Analytical Elastic-Perfectly Plastic Contact Model, International Journal of Solids and Structures, 49, pp $3129-3141$.

[9]. Stronge, W.J. (2000b): Contact Problems for Elasto-Plastic Impact in Multi-Body Systems, In: Bernard Brogliato (Ed.) Impacts in Mechanical Systems: Analysis and Modelling, Springer, Berlin.

[10]. Alcalá, J., Giannakopoulos, A.E., Suresh, S. (1998): Continuous Measurements of Load Penetration Curves with Spherical Microindenters and the Estimation of Mechanical Properties, Journal of Materials Research, 13, 1390-1400.

[11]. Bartier, O., Hernot, X., Mauvoisin, G., (2010): Theoretical and experimental analysis of contact radius for spherical indentation, Mechanics of Materials, 42, 640-656. 


\section{APPENDIX A: ALGEBRA IN THE DERIVATION OF THE CUBIC EQUATION FOR $\boldsymbol{\delta}_{\text {tep }}$}

For convenience, equation (20) is repeated here as equation (A1).

$$
K_{h}\left(\delta_{t e p}-\delta_{y}\right)^{3 / 2}-1.5 K_{h}\left(\delta_{t e p}-\delta_{y}\right)^{1 / 2}\left(\delta_{t e p}-\delta_{p}\right)=F_{\delta=\delta_{p}}-K_{h} \delta_{y}^{3 / 2}
$$

Substituting $X=\delta_{t e p}-\delta_{y}, Y=\delta_{t e p}-\delta_{p}$, and $Z=F_{\delta=\delta_{p}}-K_{h} \delta_{y}^{3 / 2}$, equation (A1) can be expressed as:

$$
X^{3 / 2}-1.5 X^{1 / 2} Y=Z / K_{h}
$$

Taking the square of both sides of equation (A2), we get:

$$
X^{3}-3 X^{2} Y+2.25 X Y^{2}=\left(Z / K_{h}\right)^{2}
$$

From the terms in equation (A3), the following expansions apply.

$$
\begin{gathered}
X^{3}=\left(\delta_{\text {tep }}-\delta_{y}\right)^{3}=\delta_{\text {tep }}^{3}-3 \delta_{y} \delta_{\text {tep }}^{2}+3 \delta_{y}^{2} \delta_{\text {tep }}-\delta_{y}^{3} \\
X^{2} Y=\left(\delta_{\text {tep }}-\delta_{y}\right)^{2}\left(\delta_{\text {tep }}-\delta_{p}\right)=\delta_{\text {tep }}^{3}-\left(2 \delta_{y}+\delta_{p}\right) \delta_{\text {tep }}^{2}+\left(\delta_{y}^{2}+2 \delta_{y} \delta_{p}\right) \delta_{t e p}-\delta_{y}^{2} \delta_{p} \\
X Y^{2}=\left(\delta_{\text {tep }}-\delta_{y}\right)\left(\delta_{\text {tep }}-\delta_{p}\right)^{2}=\delta_{\text {tep }}^{3}-\left(\delta_{y}+2 \delta_{p}\right) \delta_{\text {tep }}^{2}+\left(2 \delta_{y} \delta_{p}+\delta_{p}^{2}\right) \delta_{t e p}-\delta_{y} \delta_{p}^{2}
\end{gathered}
$$

By substituting these expansions into equation (A3), collecting like terms, and simplifying, a cubic equation in terms of $\delta_{\text {tep }}$ is obtained as shown.

$$
\delta_{\text {tep }}^{3}-\Lambda_{1} \delta_{\text {tep }}^{2}+\Lambda_{2} \delta_{\text {tep }}-\Lambda_{3}=0
$$

where $\Lambda_{1}=6 \delta_{p}-3 \delta_{y} ; \Lambda_{2}=9 \delta_{p}^{2}-6 \delta_{y} \delta_{p} ; \Lambda_{3}=4 \delta_{y}^{3}-12 \delta_{y}^{2} \delta_{p}+9 \delta_{y} \delta_{p}^{2}+\left(2 Z / K_{h}\right)^{2}$. The constants $\delta_{p}, \delta_{y}$, and $K_{h}$ are obtained from equations (16), (10), and (8).

\section{APPENDIX B: DEFORMATION WORK DURING CONTACT LOADING}

Using equations (3) - (7) the work done in each of the three loading regimes can be obtained as shown.

Work done during the elastic loading regime

$W_{e}=\int_{0}^{\delta_{y}} F_{e} d \delta=\int_{0}^{\delta_{y}} K_{h} \delta^{3 / 2} d \delta=0.4 K_{h} \delta_{y}^{5 / 2}$

Work done during the nonlinear elastoplastic loading region

$$
\begin{array}{r}
W_{e p}^{I}=\int_{\delta_{y}}^{\delta_{t e p}} F_{e p}^{I} d \delta=\int_{\delta_{y}}^{\delta_{t e p}}\left[K_{h}\left(\delta-\delta_{y}\right)^{3 / 2}+K_{h} \delta_{y}^{3 / 2}\right] d \delta \\
=0.4 K_{h}\left(\delta_{\text {tep }}-\delta_{y}\right)^{5 / 2}+K_{h} \delta_{y}^{3 / 2}\left(\delta_{\text {tep }}-\delta_{y}\right)
\end{array}
$$

Work done during the linear elastoplastic loading region

$$
\begin{array}{r}
W_{e p}^{I I}=\int_{\delta_{t e p}}^{\delta_{p}} F_{e p}^{I I} d \delta=\int_{\delta_{t e p}}^{\delta_{p}}\left\{K_{l}\left(\delta-\delta_{\text {tep }}\right)+K_{h}\left[\left(\delta_{\text {tep }}-\delta_{y}\right)^{3 / 2}+\delta_{y}^{3 / 2}\right]\right\} d \delta \\
=0.5 K_{l}\left(\delta_{p}-\delta_{t e p}\right)^{2}+K_{h}\left[\left(\delta_{t e p}-\delta_{y}\right)^{3 / 2}+\delta_{y}^{3 / 2}\right]\left(\delta_{p}-\delta_{t e p}\right)
\end{array}
$$


Work done during the fully plastic loading regime

$W_{f p}=\int_{\delta_{p}}^{\delta_{m}} F_{f p} d \delta=\int_{\delta_{p}}^{\delta_{m}}\left[K_{p}\left(\delta-\delta_{p}\right)+F_{p}\right] d \delta=0.5 K_{p}\left(\delta_{m}-\delta_{p}\right)^{2}+F_{p}\left(\delta_{m}-\delta_{p}\right)$

Using equations (B1) - (B4), the following work done can be determined.

Work done up to the end of the elastic loading regime, $W_{y}$

$W_{y}=W_{e}=0.4 K_{h} \delta_{y}^{5 / 2}$

Work done up to the end of region $I, W_{\text {tep }}$

$W_{\text {tep }}=W_{y}+W_{e p}^{I}=0.4 K_{h} \delta_{y}^{5 / 2}+0.4 K_{h}\left(\delta_{\text {tep }}-\delta_{y}\right)^{5 / 2}+K_{h} \delta_{y}^{3 / 2}\left(\delta_{t e p}-\delta_{y}\right)$

Work done up to the end of region II, $W_{p}$

$$
\begin{aligned}
W_{p}=W_{\text {tep }}+ & W_{e p}^{I I} \\
& =0.4 K_{h} \delta_{y}^{5 / 2}+0.4 K_{h}\left(\delta_{\text {tep }}-\delta_{y}\right)^{5 / 2}+K_{h} \delta_{y}^{3 / 2}\left(\delta_{\text {tep }}-\delta_{y}\right) \\
& +0.5 K_{l}\left(\delta_{p}-\delta_{\text {tep }}\right)^{2}+\left[K_{h}\left(\delta_{\text {tep }}-\delta_{y}\right)^{3 / 2}+K_{h} \delta_{y}^{3 / 2}\right]\left(\delta_{p}-\delta_{\text {tep }}\right)
\end{aligned}
$$

Work done up to a terminal point in the fully plastic regime, $W_{m}$ $W_{m}=W_{p}+W_{f p}$

$$
\begin{aligned}
& =0.4 K_{h} \delta_{y}^{5 / 2}+0.4 K_{h}\left(\delta_{t e p}-\delta_{y}\right)^{5 / 2}+K_{h} \delta_{y}^{3 / 2}\left(\delta_{\text {tep }}-\delta_{y}\right) \\
& +0.5 K_{l}\left(\delta_{p}-\delta_{\text {tep }}\right)^{2}+\left[K_{h}\left(\delta_{\text {tep }}-\delta_{y}\right)^{3 / 2}+K_{h} \delta_{y}^{3 / 2}\right]\left(\delta_{p}-\delta_{t e p}\right) \\
& +0.5 K_{p}\left(\delta_{m}-\delta_{p}\right)^{2}+F_{p}\left(\delta_{m}-\delta_{p}\right)
\end{aligned}
$$

